\title{
Human basal cell carcinoma: the induction of anagen hair follicle Differentiation
}

\author{
H. J. Morgan,1 A. Benketah, 1 C. Olivero,, E. Rees, ${ }_{1}$ S. Ziaj, 1 A. Mukhtar,, S. Lanfredini 1 and \\ G. K. Patel 1 \\ 1European Cancer Stem Cell Research Institute, School of Biosciences, Cardiff University, Cardiff, UK \\ doi:10.1111/ced.14108
}

Summary Background. Consistent with cancer stem cell driven pattern of growth, human basal cell carcinomas (BCCs) demonstrate differentiation along hair follicle (HF) lineages.

Aim. To define the pattern of differentiation and therapeutic targets that promote BCC differentiation and therefore BCC cancer stem cell exhaustion.

Methods. An alkaline phosphatase substrate kit was used to determine dermal papilla cells within the BCC stroma. Autonomous HF cycle-dependent gene expression was identified by analysis of the human homologues of a murine gene set (total 2289 genes) that is differentially expressed in hair cycle phases. The findings were validated by quantitative real-time PCR and immunofluorescence, as well as in vitro transforming growth factor (TGF)-b2 stimulation of BCC cancer stem cell colonies. Results. As in the HF, keratin expression in the inner root sheath and matrix in BCC correlated with proliferative index and was tightly regulated, despite the absence of dermal papilla cells. Cross-species microarray analysis comparing human BCC and murine synchronous HF growth cycle datasets revealed $74 \%$ concordance with telogen differentiation compared with anagen $(23 \%, \mathrm{P}<0.01)$ and catagen (49\%; P < 0.01). Incomplete anagen differentiation within BCC was characterized by reduced expression of the anagen master regulator DLX3 (_5.5-fold), and increased expression of telogen-associated genes: AEBP1 (2.2-fold), DEFB8 (35.3-fold), MMP3 (106.0-fold) and MMP12 (12.9-fold). Restoration of dermal papilla signals by in vitro addition of TGF-b2 enhanced anagen differentiation.

Conclusion. Our findings show that BCC cells differentiate along HF lineages and may be susceptible to exogenous HF cycle modulators.

\section{Introduction}

During embryogenesis, the hair follicle (HF) miniorgan emerges from a well-choreographed and coordinated reciprocal pattern of growth factor signalling between the epidermis and dermal condensate. 1 Likewise, the postnatal HF is dynamic, and undergoes cyclical growth (anagen), apoptosis (catagen) and rest (telogen) phases during the hair cycle. As in hair development, the hair cycle is also regulated by coordinated release of soluble signalling molecules from the epithelium, in particular the HF bulge and bulb, the mesenchymal dermal papilla (DP) and the surrounding fat. Alkaline phosphatase (ALP) activity is routinely used as a DP marker, and it is evident throughout the hair cycle, with expression being maximal in early anagen and decreasing in the proximal portion after mid-anagen. Transition from telogen to anagen is initiated by the secretion of transforming growth factor (TGF)-b2 ligand by DP cells, and the signal is received through the TGF-b receptor II, expressed on bulge and bulb keratinocytes.2 Through direct regulation of Tmeff1, TGF-b2 inhibits signalling of bone morphogenic protein (BMP), a hallmark of quiescence during telogen. This is followed by reduced BMP4 production by the DP and bulb, followed by increased expression of Noggin and downregulation of Bmpr1a. Simultaneously, there is an increase in BMP4 expression in the dermis.3 Anagen HF growth and differentiation is associated with an increase in soluble Wingless-ints (Wnt3, Wnt4, Wnt10a/b and Wnt11), which are released by bulb matrix cells.4 Later, accumulation of TGF-b1 and fibroblast growth factor (Fgf) 18, produced by bulge keratinocytes, induces catagen. 5,6 Accumulation of BMP2 and BMP4 from epithelial and dermal compartments contributes to HF quiescence during telogen.7 The hair cycle also defines the HF differentiation patterns, as evidenced by microarray analysis of murine HFs in synchronous growth.8,9 The fully formed HF during anagen generates the hair shaft growth, comprised of an outer root sheath (ORS), companion layer, four inner root sheath (IRS) layers and four hair shaft layers. Each HF layer has a distinct and lineage-specific differentiation pattern, which is characterized by specific keratin expression patterns. 10

Human basal cell carcinomas (BCCs) are thought to be derived from HF bulge keratinocyte stem cells (KSCs) as evidenced by the following: (i) BCC growth is frequently observed on hair-bearing skin; (ii) BCC cells resemble basal cells of the HF ORS; and (iii) the human HF bulge KSC cell surface marker CD200 is also expressed on human BCC cancer stem cells.11 Lineage tracing experiments in transgenic mouse models of BCC have shown that BCCs are derived from HF bulge KSCs and demonstrate HF differentiation.12,13 BCC growth is dependent upon and characterized by an essential dense and inductive fibroblast-rich stroma that artificially fractures during histological processing. The role of the BCC stroma, which may or may not contain DP cells, in human BCC differentiation is unknown. Further understanding of the pattern of BCC differentiation is important, as we know that promoting 
epidermal differentiation by administration of retinoids can hinder squamous cell carcinoma (SCC) growth. Therefore, in the current study, we sought to define the pattern of BCC differentiation, and elucidate potential therapeutic targets that might promote $\mathrm{BCC}$ differentiation and result in $\mathrm{BCC}$ cancer stem cell exhaustion.

\section{Methods}

BCC, SCC and normal skin tissues were obtained after approval by the UK NHS R\&D and Local Research Ethics Committee (09-WSE-02-1). Patients were recruited from Hywel Dda and Cardiff and Vale University Health Boards, and all gave informed written consent.

Tissue dissociation and in single cell culture

Tissue for cell culture was processed as previously described.14 BCC cells were plated onto a feeder layer (50 Gyirradiated 3T3 murine fibroblasts) and keratinocyte serum-free media (Gibco, Thermo Fisher Scientific,

Loughborough, Leicester, UK) supplemented with $20 \mathrm{ng} / \mathrm{mL}$ epidermal growth factor (Peprotech IEC Ltd, London, UK), $10 \mathrm{ng} / \mathrm{mL}$ FGF-2 and $0.15 \mathrm{ng} / \mathrm{mL}$ bovine pituitary extract, $25 \mathrm{U} / \mathrm{mL}$ of penicillin, $25 \mu \mathrm{g} / \mathrm{mL}$ streptomycin and 10 $\mu \mathrm{g} / \mathrm{mL}$ amphotericin (all Gibco, Thermo Fisher Scientific) was used.

Microarray

For each biological replicate $500 \mathrm{ng}$ of high-quality total RNA was labelled using an RNA kit (Illumina TotalPrep RNA Amplification Kit; Ambion, Thermo Fisher Scientific) and hybridized to a BeadChip array (HumanHT-12 v4; Illumina, Cambridge, UK) according to the manufacturer's instructions. The resulting data were analysed using the Bioconductor packages in the R statistical program for data processing for all the subsequent steps. For analysing Illumina microarray data, the Lumi package was used for assessing quality control of the samples, and removing outliers, before transforming the data using variance stabilizing transformation, and normalizing the conditional quantile normalization method. Finally, genes were annotated and mapped at the probe level using the lumiHumanAll.db and luminHumanIDMapping programs respectively.

Microarray data for the first two synchronized murine hair growth cycles were obtained from the data of Lin et al.8 via the National Center for Biotechnology Information (NCBI) Gene Expression Omnibus (www.ncbi.nlm.nih.gov/geo). Log fold changes and z-scores were generated from both the Lin et al.8 dataset and our own microarray dataset.8 The panel of 80 homologous genes of interest were compared between the two microarray datasets using the online tool Morpheus (https://software.broadinstitute.org/morpheus) to generate heat maps and to perform Pearson correlation and hierarchical clustering.

$\mathrm{RT}_{2}$ Profiler Array

RT2 Profiler PCR Array (PAHS-035Y) was undertaken to determine the expression of 84 TGF-b/BMP signalling pathway regulated genes in six BCC, three SCC and five normal skin samples, in accordance with the manufacturer's instructions (QIAGEN, Manchester, UK). Briefly, cDNA was synthesized using the RT2 First Strand Kit and mixed with the ready-to-use PCR mastermix (both QIAGEN) and subsequently added to the RT2 Profiler PCR Array plate (384-well) containing the SYBR_Green-optimized primer assays of interest. The plate was run on the QuantStudio 7 Flex Real-Time PCR System (Thermo Fisher Scientific).

Immunofluorescence labelling

Immunofluorescence on frozen sections was performed using standard techniques as previously described.14,15 For the list of antibodies used, see Table S1. Images were acquired using a camera (Axiocam; Zeiss, Cambourne,

Cambridgeshire, UK) and processed using ImageJ software (https://imagej.nih.gov/ij/download.html).

Alkaline phosphatase staining

Sections were cut on a cryostat and fixed in acetone (Thermo Fisher Scientific) for 15 min, air-dried and washed for 10 min in Tris buffer (pH 8.2). The ALP substrate kit (Vectastain; Vector Laboratories Ltd, Peterborough, UK) consisted of three reagents; two drops of reagent 1 were mixed with $5 \mathrm{~mL}$ of Tris buffer, followed by addition of two drops each of reagents 2 and 3. The sections were then incubated in the above substrate mixture for 30 min, followed by washing in tris buffer. The slide was counterstained with haematoxylin (Sigma-Aldrich, Gillingham, Dorset, UK) for 5 min and mounted.

Quantitative real-time PCR

Real-time quantitative reverse transcription analysis was performed on the QuantStudio 7 Flex Real-Time PCR System (Thermo Fisher Scientific). Total humanspecific b-actin was used as the housekeeping gene. Primer details are described in Table S2.

In vitro transforming growth factor-b stimulation

BCC colonies were established as previously described 15 and treated in triplicate as follows: fresh media and fresh media with $100 \mu \mathrm{mol} / \mathrm{L}$ TGF-b2 (Peprotech IEC Ltd). Each well was photographed under an inverted light microscope with a 29 objective lens and a digital camera system (Axiocam; Zeiss). Photographs were converted into binary images and both colony number and size were determined using Image J software (NIH, Bethesda, MD, USA). After $48 \mathrm{~h}$, RNA was extracted from each well and reverse transcribed into cDNA.

Statistical analysis

Paired t-test and Fisher exact tests were used to compare real-time PCR data and microarray concordance respectively, using GraphPad Prism software (Graph- Pad, La Jolla, CA, USA). 


\section{Results}

Regulation of hair follicle keratin expression in basal cell carcinoma is not dependent on dermal papilla Labelling of hair follicle tissue sections with versican and a-smooth muscle actin both also labelled the dermal sheath fibroblasts (Fig. 1a). The hair bulb and DP expressed CD56, BMPs and BMP receptor 1a, but these are also not specific for the DP (Fig. 1b,c). After optimizing ALP labelling in HFs (Fig. 1d), expression was determined in eight BCC samples (Fig. 1e). Although ALP accurately labelled the DP, additional positive cells arranged in linear patterns were observed within the surrounding stroma, suggestive of dermal blood vessels, both in the BCC and the normal HF tissue. Using immunofluorescence, ALP did indeed simultaneously detect DP in the HF, which remained CD31-negative, but dual positive labelling was observed elsewhere in the stroma (Fig. 2, upper row). In BCC sections, all ALPpositive cells within the stroma coincided with CD31-positive endothelial cells (Fig. 2), although not all CD31-positive endothelial cells were ALP-positive.

Incomplete anagen hair follicle differentiation in basal cell carcinoma

Of the 89 murine hair cycle-dependent genes, derived from microarray data gene sets of the first two synchronized murine hair growth cycles (Tables S4-12), 80 human homologues were identified. We found that 38 were upregulated (19 significantly upregulated) and 42 downregulated (16 significantly downregulated) within our BCC compared with the human scalp HF dataset (Table S3). Concordant gene regulation was demonstrated in 59 (74\%) of the 80 telogen genes, but only $23 \%$ of the anagen genes $(\mathrm{P}<0.01)$ and $49 \%$ of the catagen genes $(\mathrm{P}<0.01)$. Using the raw dataset, we determined and compared the log fold change and zscores for the entire dataset and the 80 human homologue gene set (Fig. 3a,b). The log fold change analysis clustered BCC with telogen (Pearson correlation coefficient 0.30), while the z-score transformed data clustered BCC between telogen and anagen differentiation (Pearson correlation coefficient_0.24). BCC genes were concordantly regulated as observed in murine telogen using a second publicly available dataset (Tables S13 and S14). Analysis of the human homologues of the telogen murine datasets identified four genes that were significantly upregulated (DEFB4, AEBP1, MMP3 and MMP12; Fig. 3c,d) and nine that were significantly downregulated in BCC, which included the anagen master regulator DLX3 (Fig. 3c) and IRS keratins (KRT35, KRT73, KRT71, KRT25, KRT27; microarray data not shown).

Induction of further anagen differentiation in basal cell carcinoma

Wnt ligand levels and Wnt-regulated gene expression were lower in BCC than that in scalp skin and SCC (Fig. 4a,b). BMP2 and -4 expression (Fig. 4c) was counterbalanced by increased expression of BMP antagonists Noggin (Fig. 4d), gremlin1 and follistatin as previously described in BCC. Equal numbers of BCC cells established over 2 weeks in primary cell co-cultures were treated with Noggin or TGF-b2 for $48 \mathrm{~h}$, commensurate with doses used to promote anagen in murine HF studies. Addition of Noggin and TGF-b2 alone or in combination with in vitro BCC cell culture, despite a relatively short half-life, was sufficient to increase DLX3 expression after $48 \mathrm{~h}$ (Fig. 4e), reversed telogen-associated gene expression (DEFB4 and MMP3, but not MMP12) and also downregulated BCC-associated expression of KRT75.

\section{Discussion}

Even though BCC growth is driven by perturbation of a single oncogenic pathway, BCC differentiation is not restricted to a single differentiation lineage, but instead appears to recreate the complex patterns observed within the HF mini-organ during anagen. In the current study, it was found that BCC anagen HF differentiation is incomplete due to: (i) absence of DP cells; (ii) low DLX3 expression; (iii) BMP expression; and (iv) low Wnt ligands and Wntregulated gene expression. This incomplete anagen is characterized by: (i) the absence of hair shaft growth; (ii) lower expression of HF-specific keratins; and (iii) expression markers of telogen AEBP1 and beta-defensin 4. We have shown that the exogenous addition of TGF-b2 further promoted anagen growth pattern in vitro, highlighting a novel approach to promote BCC differentiation that may be of therapeutic importance. These findings also emphasize the importance in BCC of epithelial mesenchymal interactions that define the spatial organization, growth and function of tissues in embryogenesis, adulthood and cancer.

\section{Conclusion}

In summary, we found that BCCs do not contain HF DP cells. Using the murine HF growth cycle microarray datasets8,9 we showed that BCCs demonstrate incomplete anagen differentiation by BCC microarray analysis, immunofluorescence, quantitative realtime PCR and in vitro-based experiments. Importantly, we showed that the exogenous addition of growth factors can promote $\mathrm{BCC}$ differentiation. These findings demonstrate a novel pattern of $\mathrm{BCC}$ differentiation that is amenable to therapeutic targeting.

\section{Acknowledgement}

This work was funded by the Ministry of Higher Education and Scientific Research (Libya) and Cancer Research UK. We wish to thank Dr Katherine Tansey and Dr Robert Andrews for their advice/data processing and analysis of the microarray data set in the Data Clinic, College of Biomedical and Life Sciences, Cardiff University. 
What's already known about this topic?

- Promoting differentiation in combination with Smoothened antagonist therapies (e.g. vismodegib) can lead to eradication of BCCs.

- Regulation of differentiation in BCC is similar to that observed in the HF ORS, matrix and IRS.

What does this study add?

- Human BCC growth and differentiation is not dependent on DP cells.

- HF differentiation in human BCCs is hair cycledependent, in which anagen differentiation is blocked and can be overcome by addition of TGFb, a known HF cycle regulator.

- There is a specific pattern of human BCC differentiation along perturbed hair cycle-dependent HF lineages, which is amenable to exogenous therapeutic manipulation.

- Using agents that promote HF differentiation may prove effective in BCC therapy, most notably when used in combination with Smoothened antagonists such as vismodegib. 


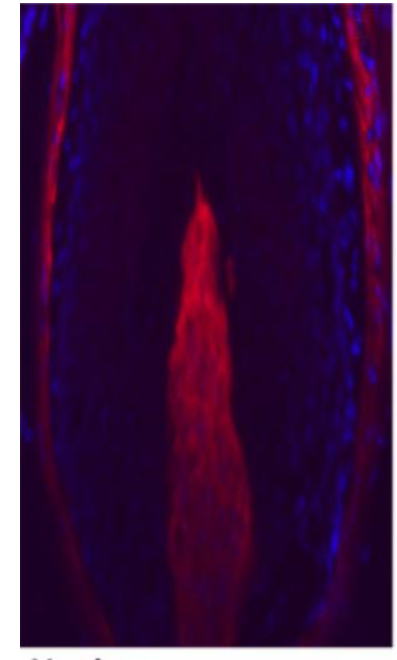

Versican

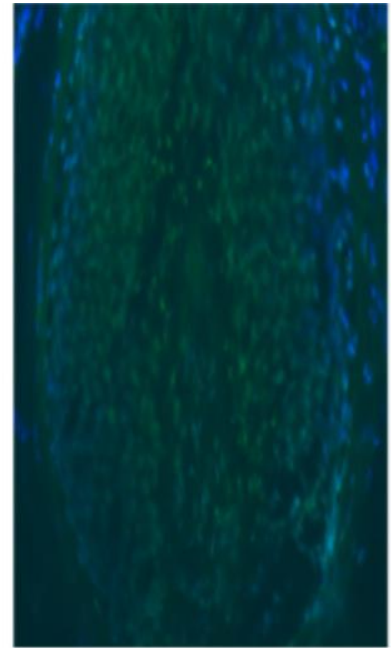

CD56

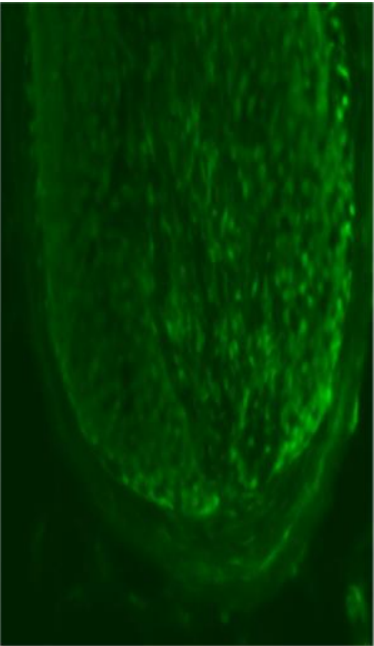

bmpr1a
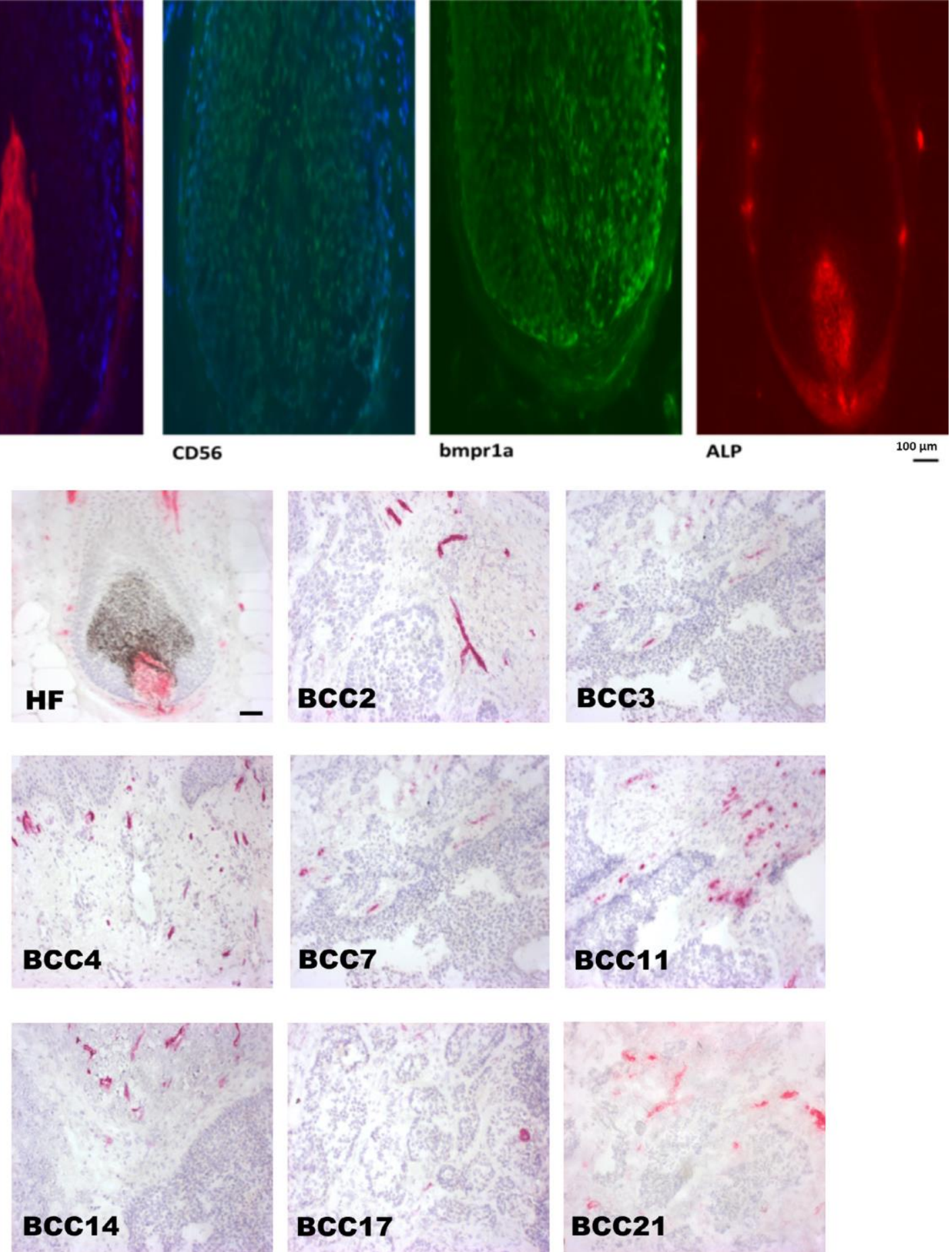

(e)

Figure 1 To identify dermal papilla by immunolabelling or staining, human scalp containing hair follicles (HFs) was labelled with (a) versican, (b) CD56 and (c) bmprla antibodies. (d) Tissue samples were also immunohistochemically stained with alkaline phosphatase (ALP). (e) Samples of human scalp containing HFs and eight sample basal cell carcinoma (BCC) tissue sections were stained for ALP. All sections were counterstained with haematoxylin. All scale bars: $100 \mu \mathrm{m}$. 


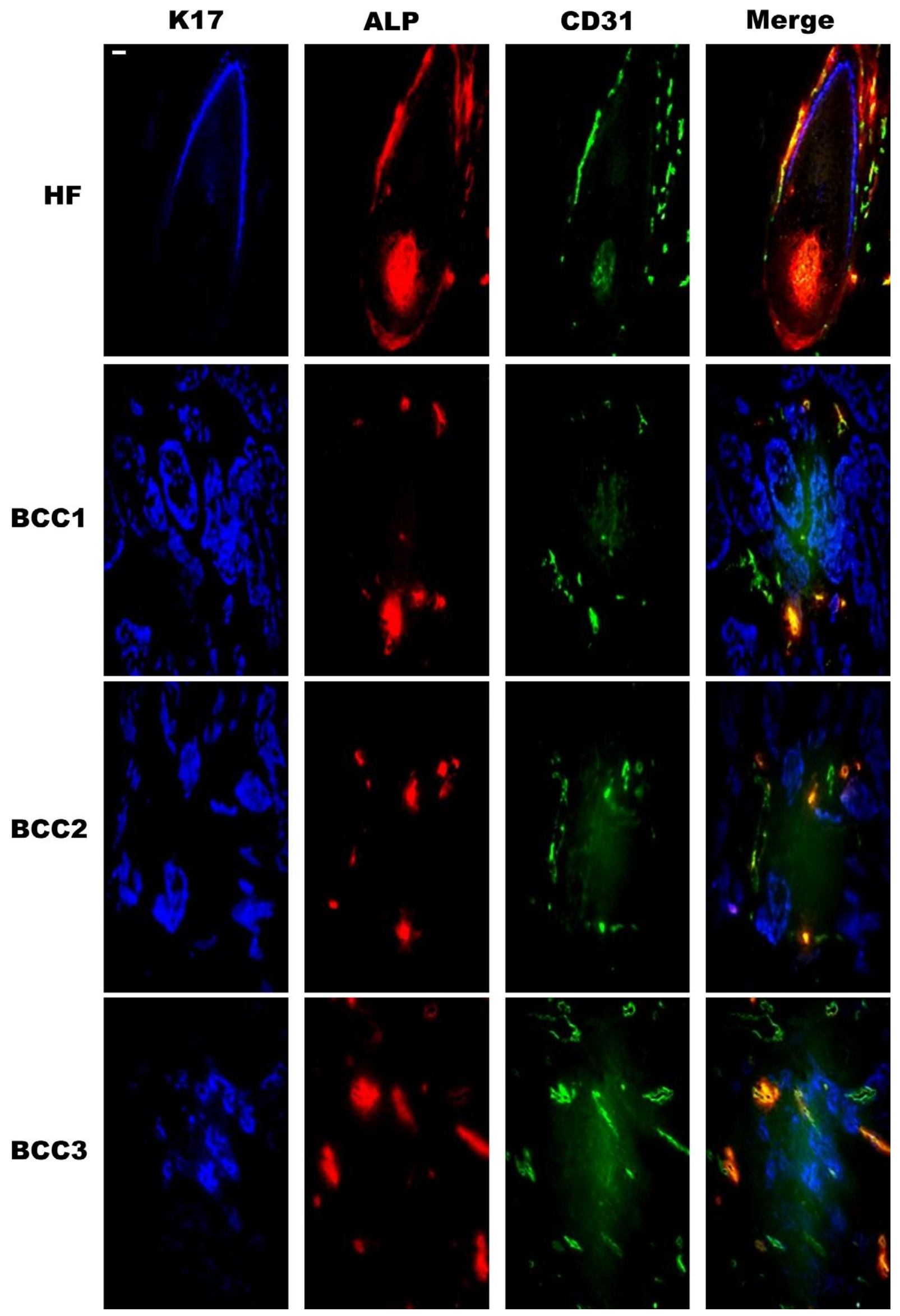


Figure 2 Dermal papilla cell labelling of hair follicle (HF) and basal cell carcinoma (BCC) tissue sections with triple-label immunofluorescence

of HF bulb and BCC tissue sections: K17 (blue), alkaline phosphatase (ALP; red) and CD31 (green). Immunolabelled tissue sections were visualized and photographed under a fluorescent microscope under 920 magnification, then processed using Image software. All scale bars: $100 \mu \mathrm{m}$

(a)

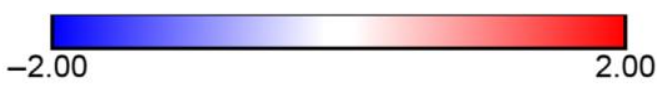

Fold change data analysis

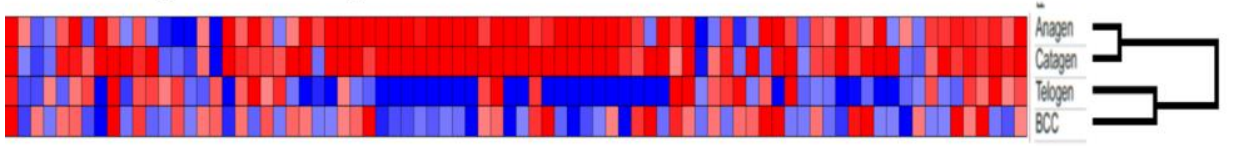

\section{Z-score data analysis}

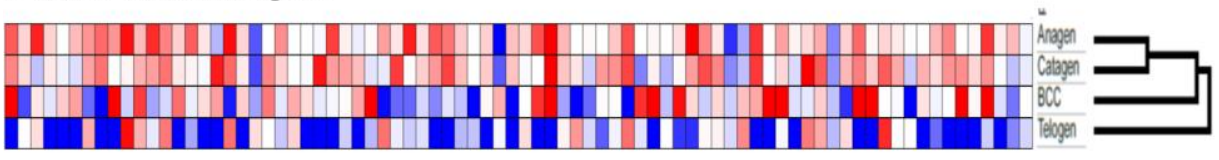

(c)

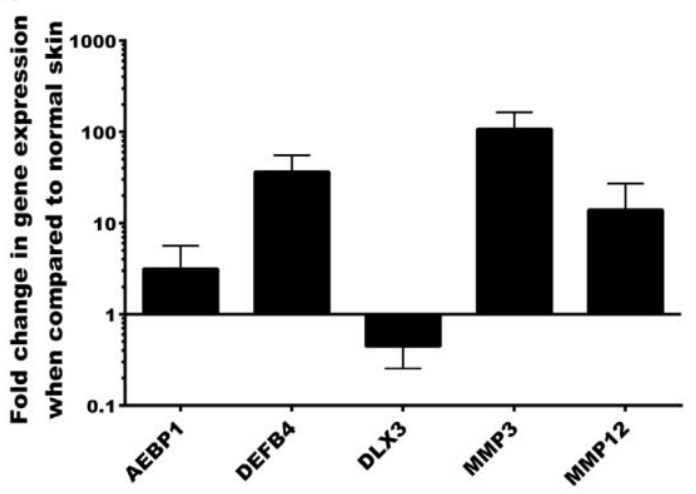

(d)

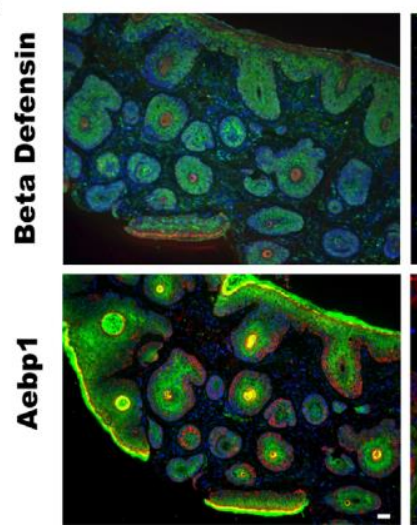

(b) Morgan $\mathrm{H}$, et al. 2019
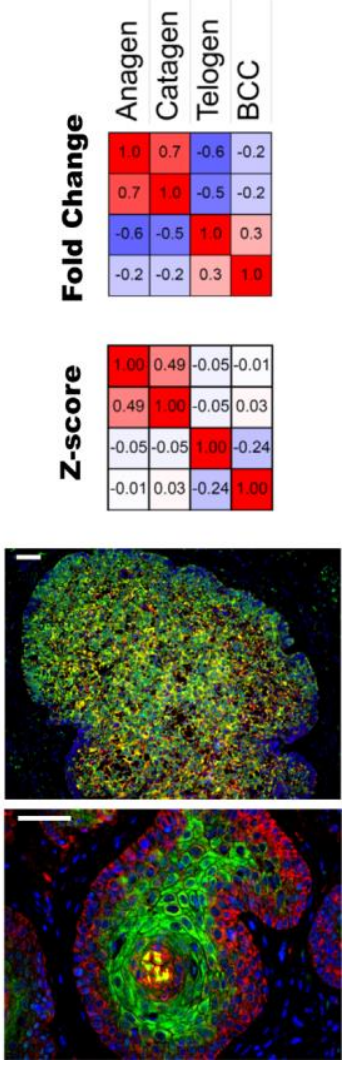

Figure 3 Basal cell carcinoma (BCC) demonstrates telogen hair follicle (HF) cycle differentiation: (a) bioinformatic microarray data comparison

of BCC and murine HF growth cycle gene set, with all human homologues $(\mathrm{n}=80)$ and $(\mathrm{b})$ BCC genes showing significant differential expression. (c) Quantitative real-time PCR analysis of BCC samples for genes overexpressed (DEFB8, AEBP1, MMP3 and

MMP12) and underexpressed (DLX3) in telogen. (d) Immunofluorescence analysis of beta-defensin 4 and Aebp1 (green) together with K14 (red) antibody-labelled BCC tissue. Immunolabelled tissue sections were visualized and photographed under a fluorescent microscope under 910 and 920 magnification, then processed using ImageJ software. All scale bars: $100 \mu \mathrm{m}$. 

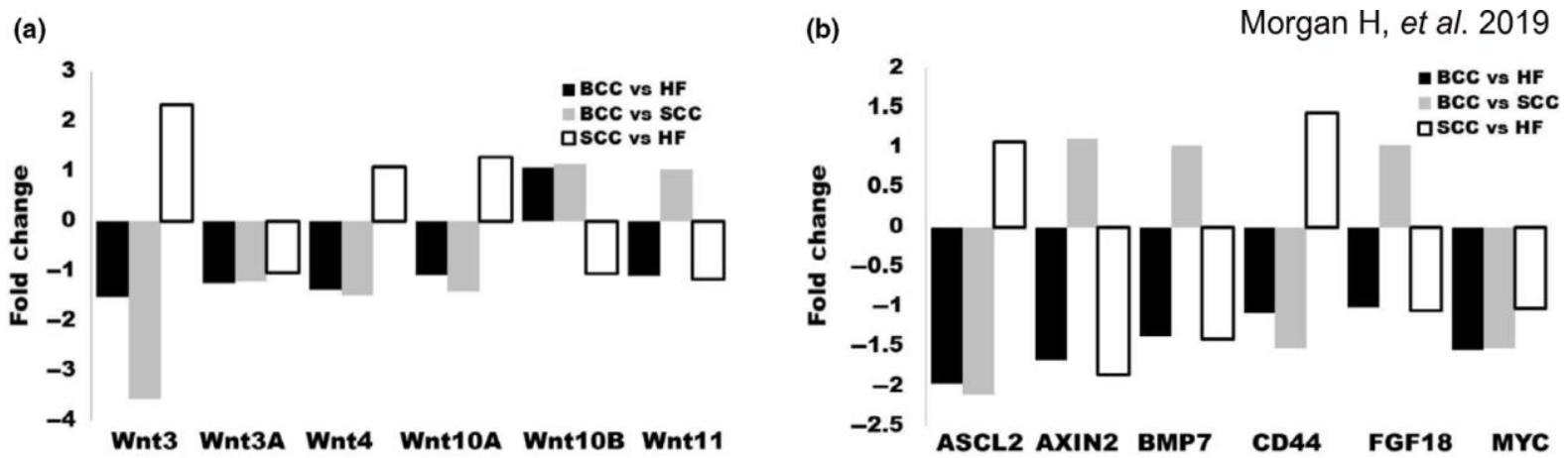

(c)

K14

BMP2

Merge
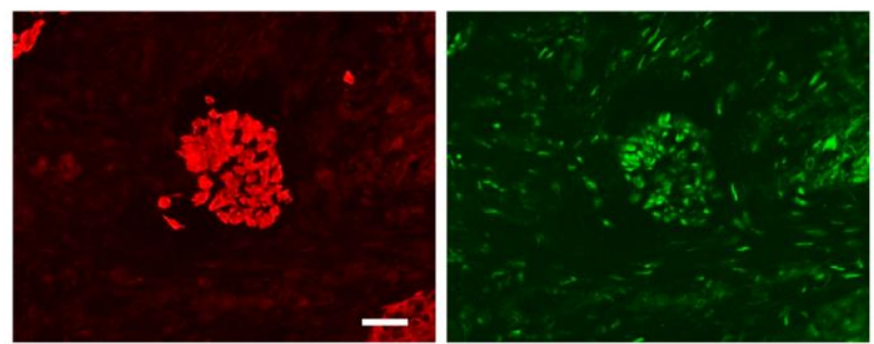

BMP4

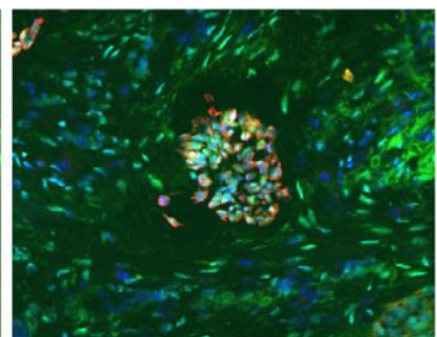

K14
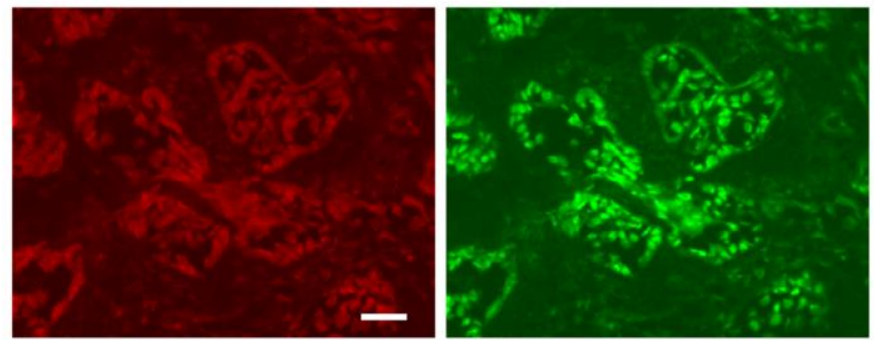

Merge

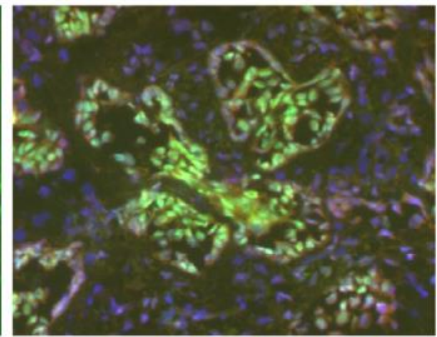

(d)

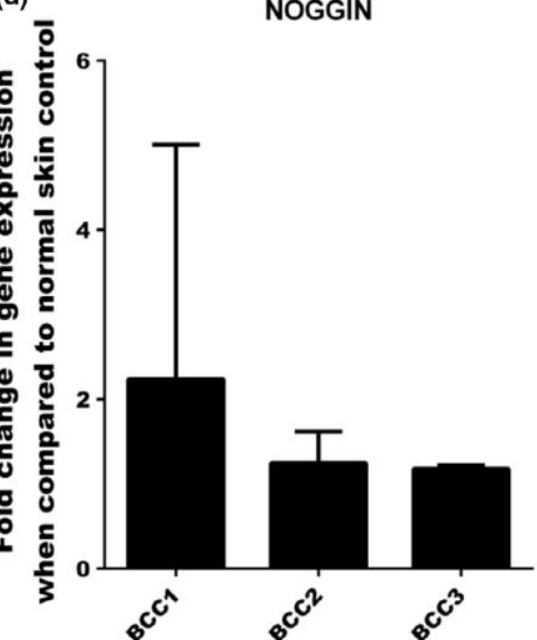

(e)

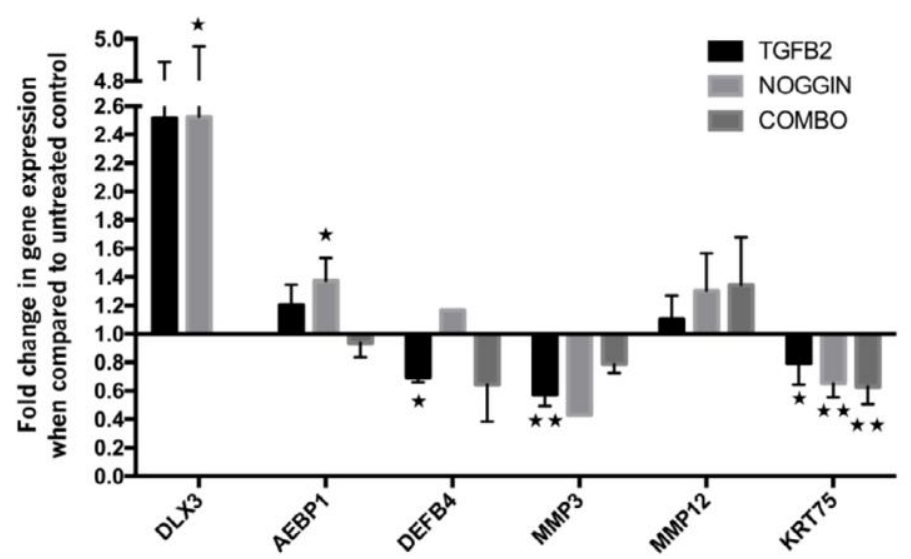

Figure 4 Induction of telogen to anagen differentiation in basal cell carcinoma (BCC): (a) microarray data expression analysis of Wnt ligands in BCC, squamous cell carcinoma (SCC) and human scalp containing hair follicles (HFs), and (b) Wnt-responsive genes. (c) Double-

label immunofluorescence of BCC tissue sections, with BMP2 or -4 (green), K14 (red) and DAPI (blue). (d) RT2 profiler тм analysis of

Noggin expression in BCC, SCC and HF. (e) Quantitative real-time PCR of human BCC cultures after treatment with Noggin, transforming growth factor (TGF)-b and combination compared with endogenous control and untreated culture. Immunolabelled tissue sections were visualized and photographed by fluorescent microscope with 910 and 920 magnification then processed using ImageJ software. All scale bars: $100 \mu \mathrm{m}$. $* \mathrm{P}<0.05$ and $* * \mathrm{P}<0.01$. 


\section{References}

1 Schneider MR, Schmidt-Ullrich R, Paus R. The hair follicle as a dynamic miniorgan. Curr Biol 2009; 19: $132-42$.

2 Oshimori N, Fuchs E. Paracrine TGF-b signalling counterbalances BMP-mediated repression in hair follicle stem cell activation. Cell Stem Cell 2012; 10: 63-75.

3 Kobielak K, Stokes N, de la Cruz J et al. Loss of a quiescent niche but not follicle stem cells in the absence of bone morphogenetic protein signaling. Proc Natl Acad Sci USA 2007; 104: 10063-8.

4 Zhang Y, Andl T, Yang SH et al. Activation of b-catenin signaling programs embryonic epidermis to hair follicle fate. Development 2008; 135: 2161-72.

5 Foitzik K, Lindner G, Mueller-Roever S et al. Control of murine hair follicle regression (catagen) by TGF-beta1 in vivo. FASEB J 2000; 14: 752-60.

6 Kimura-Ueki M, Oda Y, Oki J et al. Hair cycle resting phase is regulated by cyclic epithelial FGF18 signaling. $\mathbf{J}$ Invest Dermatol 2012; 132: 1338-45.

7 Rendl M, Lewis L, Fuchs E. Molecular dissection of mesenchymal-epithelial interactions in the hair follicle. PLOS Biol 2005; 3: e331.

8 Lin KK, Chudova D, Hatfield GW et al. Identification of hair cycle-associated genes from time-course gene expression profile data by using replicate variance. Proc Natl Acad Sci USA 2004; 101: 15955-60.

9 Geyfman M, Gordon W, Paus R, Andersen B.

Identification of telogen markers underscores that telogen is far from a quiescent hair cycle phase. J Invest Dermatol 2012; 132: 721-4.

10 Schweizer J, Langbein L, Rogers MA, Winter H. Hair follicle-specific keratins and their diseases. Exp Cell Res 2007; 313: 2010-20.

11 Colmont CS, Benketah A, Reed SH et al. CD200 expressing human basal cell carcinoma cells initiate tumor growth. Proc Natl Acad Sci USA 2013; 110 : 1434-9.

12 Hutchin ME, Kariapper MS, Grachtchouk M et al. Sustained hedgehog signaling is required for basal cell carcinoma proliferation and survival: conditional skin tumorigenesis recapitulates the hair growth cycle. Genes Dev 2005; 19: 214-23.

13 Grachtchouk M, Pero J, Yang SH et al. Basal cell carcinomas in mice arise from hair follicle stem cells and multiple epithelial progenitor populations. J Clin Invest 2011; 121: 1768-81.

14 Patel GK, Yee CL, Terunuma A et al. Identification and characterization of tumor-initiating cells in human primary cutaneous squamous cell carcinoma. J Invest Dermatol 2012; 132: 401-9.

15 Colmont CS, Ketah AB, Errington RJ et al. Human basal cell carcinoma tumor-initiating cells are resistant to etoposide. J Invest Dermatol 2014; 134: 867-70. 


\section{Supporting Information}

Additional Supporting Information may be found in the online version of this article:

Table S1. List of antibodies used for immunofluorescence.

Table S2. List of TaqMan primers used in quantitative real-time PCR.

Table S3. Validated gene set.

Table S4. Cluster 1-4: Peak early anagen.

Table S5. Cluster 5-6: Peak mid anagen.

Table S6. Cluster 7-8: Peak late anagen.

Table S7. Cluster 9-12: Drop in catagen.

Table S8. Cluster 13: Drop in catagen.

Table S9. Cluster 14-15: Peak in catagen.

Table S10. Cluster 16-18: Sharp rise in catagen.

Table S11. Cluster 19-21: Slow rise in catagen.

Table S12. Cluster 22-24: Drop during catagen.

Table S13. Genes overexpressed in telogen.

Table S14. Genes underexpressed in telogen. 